ISSN: 2638-5007

Volume 3, Issue 1, 2020, PP: 06-16

\title{
Through the Ethical Lenses: There is Really More than Meets the Eye with Covid-19
}

\author{
Fatimah Binte Abdul Lateef
}

\author{
Senior Consultant, Dept. of Emergency Medicine, Singapore General Hospital, Singapore. \\ *Corresponding Author: Prof. F Lateef, Dept. of Emergency Medicine, Singapore General Hospital, Singapore.
}

\section{Abstract}

COVID 19 represent the pandemic of the century. It challenged all of us to look at the way we live, work, practice and interact with one another. This paper looks at the ethical issues and dilemmas COVID 19 posed. It looks at the difference between the day to day practice versus working under exceptional crisis situations. Common ethical principles are discussed candidly by the author, who is a full time frontline Emergency Physician and also a Member of Parliament in Singapore. Ethical principles of beneficence, justice, equity and virtue ethics are discussed with examples, as well as the three factors which are critical in managing a pandemic: leadership, values and balancing individual rights versus collective duties and responsibilities in society.

Keywords: COVID 19, ethics, ethical principles, beneficence, equity, justice, social distancing, quarantine, rights, virtue ethics, healthcare workers, pandemic.

\section{INTRODUCTION}

There is ethics in every doctor-patient interaction. There is ethics in every case a doctor handles. It may not always be obvious that ethics is involved in every step but it is central to our 'duty to care' to patients.

Ethics may be the last thing on the minds of people during crisis and pandemics. It is also not the most obvious element, given the more overt and pressing issues on hand. However, ethics is central to the multiple, complex decisions made during a pandemic. It is entwined in our workflow processes, triage, notification processes, quarantine order and decisions, case definition, identification and management. There are no easy answers for ethical issues during pandemics and epidemics. Ethical principles may help to explain why doctors and healthcare workers (HCW) do the work they do; spending long hours in complex workflows, managing very ill patients and even risking their own safety and lives, in some cases. They may not even have time to pause and ponder during pandemics, knowing that their duties have to be executed, as a part of their professional code of conduct.
Ethics refers to the judgement about what is right or wrong, or what is worthy of praise or blame, to put it simply. Medical ethics involve examining a specific problem or clinical case and then utilizing values, medical facts and logic to decide on the best course of action. Bioethics is a subset of ethics that incorporates ethical principles for real or potential ethical dilemmas facing clinicians in practice. A code of ethics provides a guide for ethical reasoning made by doctors. It helps guide the framework on treatment of individual patients. This, in general, may help set the standards for healthcare workers (HCWs) and dispel confusion and uncertainty, pertaining to managing patients, patients' rights and also responsibilities. It represents one of the major pillars of medical practice and inadvertently, it will help to reassure the public with the trust they have invested in HCWs. Doctors and HCWs come into a contract when they selected the profession to go into. It is a contract to society, with a duty to treat and a duty to care with strong dedication. (1-5)

Virtue ethics, on the other hand, is a generic term that emphasizes physicians characteristics more than just doctor-specific action. These characteristics include 
virtues such as compassion, integrity, fortitude, fidelity, temperance, kindness, fairness and even self-effacement. Altruism and dedication are also on the list. All these are very closely linked to medical professionalism. $(2,6)$ In general, ethical guidelines must be contextualized in order for it to make sense and be of practical value to practitioners of medicine and healthcare. Values like respect for all persons and acting with their best interest in mind, maintaining confidentiality, understanding autonomy of patients' decision, declaring conflicts of interest, maleficence and beneficence have various modes of execution and application.(2) Culture too may have some bearing on how ethical principles are executed, based on the local context. On a bigger picture, there are also issues such as allocation of limited resources, equity and distributive justice to be considered. Whilst having all these ethical principles and framework available for our reference, ethical challenges and dilemmas are still common in the face of our practice of medicine. This is probably because medicine deals with humans, human sufferings and diseases.

\section{The Covid-19 Pandemic}

In early December 2019, cases of persons with pneumonia of uncertain etiology began to surface in Wuhan, Hubei Province, China. Many of them were linked to a seafood wholesale market, selling Huanan delicacies. This is a market popular with the sales of live animals, which is very commonly found in this part of China. Other sources of the potential links were still being investigated then. A cohort of these patients developed severe acute respiratory distress and some rapidly developed acute respiratory distress syndrome (ARDS) and respiratory failure. By early January 2020, a novel coronavirus was identified by the Chinese Communicable Diseases Centre (CDC) and this was then subsequently named the COVID-19 by World Health Organization (WHO).(7) By then the numbers affected across many nations outside China, began to increase. The numbers of death within China itself increased exponentially. The global community watched anxiously as reported numbers climbed and countries began to execute border checks, travel restrictions, temperature monitoring, screening questionnaire and other restrictions protocols. Wave after wave, region by region, COVID-19 seems to spread incessantly. The numbers of death also plummeted. By then with the spread to over 190 countries, it had reached pandemic levels, globally. In healthcare institutions, concerns loomed as to whether the world would see another infectious disease epidemic similar to SARS (Severe Acute Respiratory Syndrome), or something worse. There began the heightened awareness and concerns due to the interdependence in the world today. $(7,8)$

By $1^{\text {st }}$ April 2020, COVID-19 had spread to over 200 countries. After the wave in China, other countries, including Europe and USA began to seek their first wave of cases increasing at an exponential rate. The number of positive cases as well as number of critically ill patients sky-rocketed and began to overwhelm many healthcare systems and countries' infrastructure, outstripping their healthcare capacity and capabilities, in many places. For all countries affected by the pandemic, everyone was learning as they go through the outbreak and as it progresses and unfolds. Scientists, researchers and medical personnel continue to make observations along the way and sharing the information with the global community. This sharing is so important as it will help translate some of these observations into effective, practical plans, policies and interventions by countries. In this context, infectious diseases outbreaks represent one of the rare times when scientists and researchers can study a disease in situ.(7)

\section{Difficult Decisions, EThical Dilemmas}

In the normal day to day care of patients, doctors and healthcare workers (HCWs) are patient-centric in their approach to care. They work on the principle of doing the 'best for every patient'. A good guide is sometimes to treat every patient as though they represent a family member. This is also individually driven and focused for each patient, who is unique. (Table 1) In disasters and crises, this principle changes to 'saving the maximum numbers of lives and limbs', within the limits and capabilities of scarce resources, manpower and healthcare infrastructure available, at that time. This aligns more with public health ethics and is thus public-centric, thus issues such as equality of persons, equity of distribution or risks and benefits to society becomes more prominent in discussion. With a pandemic like COVID-19, the shift from one principle of care to the other may create conflicts, stress and tensions for HCWs, under these emergency conditions. $(6,9,10)$ Thus, it may surface multiple issues pertaining to uncertainties on how to proceed, with the overwhelming numbers 
Through the Ethical Lenses: There is Really More than Meets the Eye with Covid-19

and needs. Moreover, in public health emergencies and pandemics like COVID-19, the massive surge in healthcare needs and patients numbers pose physical and psychological challenges. In the management of the more critically ill patients who are infected, there may not be the feasibility and possibility to discuss with the patient, his family members, or even clinical ethics teams on the preference and values pertaining to each individual patient. This is a challenge public health crisis puts upon us. This is the limitation we face in public health emergencies. There must probably be a balance between individual and group needs.(Table 2) However, the question is, where do we draw the line knowing that in pandemics limited resources may not enable us to save lives, the way we do in normal, day to day conditions? $(6,11-16)$

Table 1. General Framework to approach a Clinical Ethical Dilemma in Day to Day Practice

\section{The following steps can be utilized:}

1. What is the problem or dilemma?

2. Gather all the relevant medical facts

3. What are the concerns, values and preferences of the medical team/ doctors?

4. What are the concerns, values and preferences of the patient/ family?

5. What are the ethical issues?

6. What ethical principles are at stake or under consideration?

7. Are there practical considerations which need to be addressed?

8. Are there psycho-social issues to be considered in the case?

Table 2. Differences between "Day to Day" Public Health and Public Health during Pandemics

\begin{tabular}{|l|l|}
\hline Day to Day Public Health & Pandemic Public Health \\
\hline Smaller scale and more manageable & Bigger or mammoth scale and greater complexities involved \\
\hline Usually less time dependent & Under a lot of time constraints and pressure \\
\hline May be part of routine healthcare management & Laced with climate of fear and uncertainties \\
\hline $\begin{array}{l}\text { Usually no issues with resources, except in } \\
\text { developing and third world countries }\end{array}$ & Limitation of resources and may require rationing \\
\hline Based on existing regulations and framework & $\begin{array}{l}\text { There may be newly implemented regulations and practice, } \\
\text { over and above the existing ones }\end{array}$ \\
\hline $\begin{array}{l}\text { Same standards of access as in usual practice in } \\
\text { the country }\end{array}$ & $\begin{array}{l}\text { Access may be a major consideration due to overwhelming } \\
\text { numbers of casualties and patients }\end{array}$ \\
\hline $\begin{array}{l}\text { Social issues remains status quo (depending on } \\
\text { the country) }\end{array}$ & $\begin{array}{l}\text { May exacerbate social crisis as both are strongly inter- } \\
\text { twined }\end{array}$ \\
\hline $\begin{array}{l}\text { Usually, in most cases, able to meet the needs } \\
\text { With infectious diseases pandemic, new challenges to } \\
\text { meet healthcare workers needs eg PPE (gowns, gloves, } \\
\text { appropriate masks, face shields). Tremendous increase in } \\
\text { needs and demands }\end{array}$ \\
\hline
\end{tabular}

Dilemmas arise from issues such as overwhelming numbers of patients, the inability of healthcare systems to cope and lack of manpower. The ultimate ethical question is how to allocate scarce resources in this pandemic and maintain morale, professionalism, conscience and ethical principles? Who gets the available intensive care unit beds, who gets a bed in hospital and who gets sent home and are there enough personal protective equipment ( PPE) for the frontline healthcare workers to perform their jobs adequately? HCWs are in a unique position in pandemics. They have a duty to care for patients, carry out their work and society look towards them to continue to serve. They each have an expertise, which is necessary, to help people get through the pandemic. With their expert skills and medical knowledge, it is hard to replace them. They must be supported so that they are able to carry out their work effectively and safely. Their well-being must be guarded as they carry out their professional duties, given all the potential risks and danger to their lives. In this context they are indispensable and must be given the respect they deserve. $(13,17,18)$ 
Through the Ethical Lenses: There is Really More than Meets the Eye with Covid-19

\section{ETHICS IN EMERGENCIES}

Ethical decision involve making hard choices. On a day to day basis, this is already challenging and can be emotional. With a pandemic situation, the stresses are multiplied many times over and ethical issues may become more prominent due to scarcity of resources aND greater awareness amongst people. There may be more demonstration of kindness, courage and selflessness compared to during normal days. People may also become more reflective during these periods. The emergencies also demand exceptional tasks from particular groups of people such as healthcare workers, transport workers, food delivery industry and cleaners.

The issues encountered during COVID 19 which surfaced ethical considerations include:

1. The decision for declaring it as a pandemic

2. Distribution of resources and management of scarce resources

3. Data collection and maintenance of confidentiality

4. Contact tracing from index cases

5. Diagnosis of super-spreaders, which some feel may be labelling people inappropriately

6. Use of certain equipment eg. Thermal scanner and temperature taking ( some feel this is an imperfect test but when used, can affect the liberty of individuals)

7. Isolation and quarantine order decisions

During the COVID 19 pandemic, I found the following factors crucial in the decision making process: (18-21)

\section{Leadership}

Leaders at the frontline involved in decision-making must have a good depth of understanding of the current situation, its potential projected progress and the upstream issues associated with it. This may be obtained by garnering help of experts on the subject. In Singapore the inter-ministry COVID 19 taskforce was formed early. In other countries, similar committees with the dedicated function of looking into the multiple issues related to COVID 19 were also quickly established. With facts and evidence, decisions, policies and frameworks can be justified and backed up. Decisions that involves ethics are usually more challenging. The moral vision must be clear in order to act appropriately, with integrity. The leader in this situation must support and standby his employee and staff. Difficult policies may inconvenience people but they must be executed, in the name of upholding the good for the maximum number of people. Leaders will have to explain these using the balance between protection versus disruption. With COVID 19, we saw how infection rate exceeded treatment capacity in many countries. This called for strong, adaptive and innovative leadership to plan alternative arrangements, external sources of support, assistance and other means to handle the load. Leadership is also responsible to ensure sufficient and appropriate public health laws as well as the participation of the country in global surveillance efforts

During the public health emergency itself, the leadership must institute multiple mandatory interventions, which may represent difficult but necessary decisions. During the COVID 19 pandemic, there were the issues of quarantine, stay home orders, leave of absence from work, testing criteria and even 'lock-down' in some countries. Leadership in institutions must align with the leadership decisions by the federal governments. In these situation, some individual liberties and autonomy have to be over-ridden in line with mandatory public health measures and may even be enforceable by action. The other important and critical issue is leadership communications. This must be timely, authoritative as needed, open, transparent, inclusive, consistent and delivered with accountability. Building trust between governments, leadership and the people is critical during these outbreaks.

\section{Balancing Individual Rights Versus Collective Duties in Society}

In crisis, some rights may have to be given up, in order to help others. This is aligned with the principle of public health. The vulnerable, the young, the elderly and sick must be protected. Some groups may pay a higher price during the pandemic than others. That is the inevitable. Practically speaking, there is no possible way to treat every individual as equally as the next person during a pandemic. The best possible approach would be to respect individuals patient rights, maintain confidentiality and treat different groups as equitably as one possibly can, based on their individual merits and pointers. (Table 3) Here again, there must be weightage of the benefits/ risks to the population versus the well-being of the individual patient. (21-25) 
Through the Ethical Lenses: There is Really More than Meets the Eye with Covid-19

Table 3. Multifactorial Considerations in Decision-making for Rationing during Pandemics based on the Principle of Doing the Greatest Good for the Greatest Number of People.

1. Considerations for existing co-morbidities eg. Stage 4 New York Heart Assn Congestive Heart Failure, Advanced Chronic Obstructive Lung Disease, Advanced stage cancer, those on palliative care, severe cognitive impairment ( Please note \# just using this criteria in isolation is ethically flawed, violates the principle of justice and increases discrimination)

2. The long term prognosis for the particular patient (no matter what condition he/ she has)

3. Functional status of the patient

4. The survival to discharge likelihood. Who is most likely to survive the hospitalization, get better and be discharged

5. Number of year of life potentially saved

6. Life cycle principle: Consider the stages of life a person has gone through ie childhood, adulthood, middle aged and senior or elderly. Priority might be given to the younger group in view they have the least opportunity to live through their life cycle stages, ie they have lived the shortest

7. Consider the instrumental value of the person in saving others, eg. a HCW may be higher on the priority list as he / she has the potential to treat others, save lives and manage diseases in patients

\#To Note: Sex, race, age, religious denominations, wealth, socio economic status are not factors of consideration.

\#\# To Note: These factors are part of a proposed multi-faceted framework for consideration

\section{Values}

During a global pandemic like COVID 19, everyone is a first responder. Every individual must comply and do their part. Values such as courage, kindness, being humane and demonstrating social acts of graciousness are important. The pandemic serves an excellent platform for reflection of our behavior and principles. People become more civic conscious and are more aware of the things that matter most in their lives. Respect for persons should be applied to all aspects of practice. This means upholding their autonomy, privacy, confidentiality and respecting their religion and culture. $(25,26)$ As we discuss about the values of the doctor and HCWs, patients' values are also important and should not be overlooked. With these in mind, the principles of beneficence (fundamental ethics of promoting good) and nonmaleficence (not doing any harm, preventing harm and removing harmful conditions and situations) are definitely relevant and applicable. $(2,25,26)$ In pandemics what touches our values to the core is when we have to decide on withholding or withdrawing medical treatment and their justifications. These would involve some of the toughest decisions doctors have to make in their lives. Withholding further treatment is usually a medical decision and done quietly, often without inputs from patient or their surrogate decision makers. Withdrawing is more obvious and involves active discussions with the next of kin. As doctors, we may have to withdraw or withhold treatment but we must never withdraw or withhold care for our patients. $(11,21,25)$

When touching on values, one that I feel strongly about is generosity, in sharing, in order to benefit as many as possible. In fact during COVID 19, sharing of observations and information amongst doctors, scientists and countries is relevant and important for all of humanity. Benefits of science are shared across the globe in the hope of positive impact for justification of the benefit sharing. As we share benefits of broad based research and preliminary findings, a word of caution on maintaining confidentiality to prevent misinterpretation or abuse of data.

The other question to ponder on is who owns these data that is being shared; is it the country, the patients, the healthcare providers or the institution?

\section{Distributing Scarce Resources}

Resource allocation takes place at different levels. For the highest level (macro level), the federal government will decide on how to divide these between various sectors such as the healthcare sector, educational sector and others. This can be planned at the prepandemic or preparedness stage and executed during the pandemic. At the institution level (meso level), it will be decided how the resources will be distributed to the various services or departments. An example would be how a hospital will allocate the masks and PPE to the different departments such as the 
Emergency Department, Intensive Care Units, general wards and others. Further on, at the micro level, departments may decide how to allocate resources to individuals such as the staff (doctors and nurses) or the patients who attend the service.

During COVID 19 we often hear of overwhelmed healthcare systems and scarce essential resources. The general principle utilized in the setting of scarcity is usually to maximize the benefits that can be produced by the scarce resource. This calls for highly responsible stewardship by the people who decides on the resource allocation and utilization. It is about who gets what and why. This is about the principle of utility, which requires allocation of resources to maximize benefits and minimize burden. For actions, utility means the actions are upright and acceptable, and they promote the wellbeing of individuals and communities. These must be reflected in our policies, enforcements, border controls, triage and management protocols during COVID 19. Equity on the other hand means the attention to fair distribution of benefits and burdens. It is challenging to always fully achieve both utility and equity and to date, there is no fixed recommended formula to resolve this. The most practical approach is to make decisions which are inclusive, transparent and accountable, with the local context borne in mind. With utility, a guide is that the outcomes will help improve welfare eg. number of life years saved or better cost-benefit ratio. $(14,21,25)$ For equity, consideration should be given to the vulnerable group as defined in the local society or community. The mask distribution by the government in Singapore during COVID 19 can be used to illustrate the principle of utility and equity. During the early phase, every household was allowed to collect a pack of 4 surgical masks from the community clubs in each constituency. However, later in the pandemic, when new evidence surfaced on the use of masks, citizens and permanent residents were allowed to collect one non disposable, reusable masks. The same principles apply in the access to healthcare in Singapore.

The other ethical term often referred to in rationing and allocation of resources is justice. Social justice is about protecting the vulnerable. Procedural justice refers to the fair process of making important decisions. Another often forgotten matter in pandemics, is that there are also the "usual" patients who need treatment. They would be those with cancer, heart failure, asthma, injuries and other problems who also need the attention of the HCWs. These patients are competing for some of the same resources as patients affected by the pandemic. This is also what poses the challenge whereby HCWs have to transition from day to day, individual patient-driven care principle to that of crisis-driven, "maximum numbers of lives saved" thinking. The latter is about doing the greatest good for the greatest numbers of patients. This often requires doctors to make the decision based on the potential of the highest numbers of lives saved and the longest survival potential.

No one principle/ factor only should be applied for each patient. Each patient must be given multifactorial consideration based on a multi-faceted ethical framework or model for decision.(Table 3) Moreover some of the more ethical decisions should not be left to individual doctors to make during crisis and pandemics. The emotional burden in such cases can be tremendous, long lasting and challenging for the individual doctor. It would be better and more practical to have guidelines and a framework, which has been decided upon by a team of inter-professional experts and contributors. This would represent a collaborative decision tree made by doctors, nurses, ethics experts, epidemiologist and other relevant persons, to be applied in the institution of practice. This way, we can elevate the individual physician from the lasting emotional burden of making the decision alone, during these challenging times. Doing this can be amounting to practicing proactive ethics, which involves planning, managing and changing ethical guidelines and regulations under which emergency HCWs operate. All the stakeholders with vested interest in an equitable solution would come to the table to discuss and reach a consensus. Proactive ethics is often practiced and applied in the public policy realm. One other principle often discussed in this context is the 'fair innings principle', whereby young children and the elderly are given priority. There are two schools of thought pertaining to this, where one agrees with it as the group may represent the vulnerable population. On the other hand, some question whether this principle is ethically permissible. $(20,21,25)$ The best approach I feel would be to relate this to other prioritization principle and factors to be considered during the distribution of scarce resources during the specific pandemic or crisis. Again, the multifactorial approach is taken here. When doing this, do bear in mind that the local culture is also important; for example: filial piety 
Through the Ethical Lenses: There is Really More than Meets the Eye with Covid-19

in Chinese and Asian societies, the value of children in that particular culture etc.

\section{HealthCare Workers (HCWS) Providing CARE IN THE TIME OF CRISIS}

HCWs have the capability and expertise to provide care and thus they have an obligation. They are the holders of privileged knowledge and public trust. Doctors who freely choose a career in Medicine is devoted to caring for the ill and sick. Their fiduciary relationship with patients they treat is part of their mutual agreement to serve and demonstrates altruism in society. Therefore they have assumed the risks and are aware of these risks. The profession can also be assumed to have a social contract with society during times of emergencies and crises. This essentially is their 'duty to care'. Beneficence represents a moral obligation of these HCWs, to further the cause of caring for patients and looking after their well-being. It is a fundamental and foundation of the patient-doctor relationship. Do HCWs have the moral duty to stay and manage patients in the face of risks or are such decision and choices based on individual staff's conscience? Beneficence, in the broader sense is also about other frontline workers carrying out their duties; such as police officers, border security workers, cleaners and transport services. It is the social contract between these professions and society. (27-30)

During pandemics, HCWs face unprecedented demands on their skills, expertise and commitment. Over and above this, they are exposed to serious risks and potential morbidity and even mortality. Its an occupational risk, and yet we have seen over and over again how HCWs worldwide go beyond their call of duty, perform professionally and admirably despite stresses and personal risks. This is ethics of virtue (discussed earlier) in physicians.(2) This also underscores the importance of inculcating these virtues into the next generation of healthcare providers and to reiterate to them that their chosen profession does come with various risks attached. This will hopefully presuppose an explicit acceptance of such hazards amongst the younger generation professionals. It must not be forgotten that they too have fears, worries, concerns, family, which they have to balance with the call of duty, obligation to patients and serving during a pandemic. HCWs do know that if they refuse to treat and manage certain cases, then it is their colleagues that have to do this on their behalf. So, is there really an unlimited duty of HCWs to provide care in any circumstances?
If there are limitations, then where exactly do they lie and should these considerations be codified into some policy or a professional code of conduct? (31-35)

The COVID 19 pandemic saw many of our young medical officers and residents going through this experience for the first time in their careers. They are now exposed to the challenges of

1. rationing of limited resources in healthcare

2. face to face encounter with a serious danger to their lives and

3. restriction of some individual movements and liberties amongst others.

In the government's plan for pandemic and emergency preparedness, it is assumed that there would be fullfledged response and cooperation from all medical staff and HCWs. But can this be assumed and how true is it? HCWs do have concerns in caring for infectious and contageous patients. They had seen deaths of HCWs during SARS and understand the threat to their personal safety. $(3,5,31)$ Beyond this, HCWs are also concerned about their own family members, including children and elderly relatives at home. As they have to execute their moral obligation to care for patients, even in pandemics, they may face ethical dilemmas. Doctors for example, know their patients' interests take precedence over their own self interest. They tend to stay and treat patients despite personal risks. This decision is made because:

a. this is their duty of care, aligned with their professional code of conduct. Its their professional duty of beneficence

b. the great, overwhelming needs of patients during these times

c. the possess the specialized skills and training of their discipline

d. the societal support and trust for HCWs and thus, a duty to accept a fair share of risk that comes with their jobs

e. they understand the utilitarian approach to do the greatest good, in the given circumstances

In return for all the above, the Ministry of Health, the institution and healthcare employers have an obligation, must support and ensure doctors who perform and work during pandemics and emergencies, are provided with at least the minimum standard of 
Through the Ethical Lenses: There is Really More than Meets the Eye with Covid-19

adequate protection to ensure their safety eg. with personal protective equipment (PPE) and for their operational needs. They must be educated about the risks and methods of transmission. Their concerns about carrying out their duties during these times are real and not just theoretical. Institutions must stand by them, support them and also ensure they understand the risks associated with their decision to stay. Their psychological wellbeing, safety and health must also be maintained and maximized. This would also mean they will be given access to healthcare if they do contract the infection or get ill. This goes for access to vaccines as well, when one has been developed. This way there will be trust between the institution and the HCWs they employ.

In Asia, there is also something called "face", whereby one would lose face if he does not continue to perform his duty like the rest of his colleagues. This is similar to peer pressure. How willing HCWs are to work depends on several factors and on their belief in the effectiveness and availability of infection control methods and PPE. This is linked to their perception of an effective mechanism and system being in place in the institution. $(3,5)$ Issue pertaining to legal protection for the HCWs during pandemics must also not be overlooked as in such circumstances they are put in a position whereby they have to make difficult decisions on triage, care issues, withholding and withdrawing of treatment or even provision of treatment beyond the pre-existing standards of care. They should thus have some avenue to be protected from inappropriate penalties. The best suggestion for this would be to come up with policy during the pre-pandemic or preparedness stage, where the HCWs themselves brainstorm and contribute to this discussion on policy.

Society too will have an ethical responsibility towards HCWs. This will likely be in terms of social recognition and maintaining their self esteem. They should be recognized for their sacrifices and contributions, yet it is not uncommon to hear of them being shunned in public places, on public transport, at food and beverage outlets because they are thought to be infectious or are carriers of the infectious agents. This misperception will have to be corrected via public education and campaigns.

\section{THE ETHICS OF QUARANTINE, ISOLATION AND} Social Distancing

Quarantine is the restriction of movement of persons who have been exposed to or might have been exposed to a communicable disease or infectious agent. People in quarantine usually do not pose high risk to the community. Isolation refers to the separation of a person who has been infected by a communicable disease, whilst he is in the period (incubation period) whereby he can spread it to others. The person who is put into isolation is at risk to the community and people around them. Both quarantine and isolation pose numerous considerations for the party that is enforcing or carrying it out as well as the people affected by the decision. The challenges can be multifaceted; logistical, operational and psychological

With COVID 19, we saw numerous stages of social distancing interventions executed in various countries, during the differentwaves of the spread of the infection. This was evolving as the pandemic progressed. Much as we usually would like to have scientific support and evidence with interventions, during the course of this pandemic, we noted that it was necessary to apply some of these interventions even with little scientific evidence or using expert observations only. Examples of social and safe distancing steps taken include:

a. isolating infected individuals

b. quarantining contacts of affected individuals ( via contact tracing)

c. curbing the size of public gathering and events

d. closure of schools ( pre-school, primary and secondary schools and institutions of higher learning)

e. restricting access ot public venues except for those offering essential services such as healthcare facilities, supermarkets and markets

f. enabling work from home and 'stay home notices'

g. limiting travels within and outside the country

These interventions are liberty limiting and social distancing actions. It may appear to be restricting personal freedom which we all value. It can sometimes be seen as intrusive and limiting individual's decision 
Through the Ethical Lenses: There is Really More than Meets the Eye with Covid-19

making capacity. Therefore any implementation must be reviewed carefully and justified. As such, we would have noted how countries introduced these measures in stages, as new observations and scientific inputs began to be put forth. The restrictions can be viewed as legitimate if in the context the affected individual is not isolated, many others can be affected and turn ill. Failure to implement these measures may result in grave harm to the public and the function of society. These restrictions are necessary for the common good and health of the society. There is one other principle that had to be applied during COVID 19. This is the Siracusa principle which is a framework for evaluating the appropriateness of limiting certain fundamentals of human rights in emergencies. These restrictions must be deemed strictly necessary as no other means are able to achieve the same outcomes or results. It should be based on scientific principles, evidence and not just some arbitrary decision which is unreasonable or discriminating. It should also respect human dignity.(36) Examples of this during the COVID 19 pandemic would include the 'lock downs' or restriction in movements.

In Singapore, as these interventions were introduced, the Prime Minister of the country himself made public statement to explain and clearly inform the public. This builds trust and enhance the buy in, especially with the open, transparent and accountable communications style. At all times, privacy and confidentiality must not be breached through the information shared. The restrictions on personal freedom and movement must also be applied equitably, with the avoidance of stigmatization of any person or group.

Good and effective communications during the pandemic response phase is crucial to counteract rumors and dampen panic and misinformation. Transparency in communications by the leadership or government is the ethical thing to do. With COVID 19, some of the information may not be concrete as they were still evolving but it is appreciated if leadership can

a. acknowledge the many uncertainties, and share the current evidence

b. provide advice and examples to illustrate clearly and

c. provide reassurance and follow up as needed, such as a hotline number to call.

\section{CONCLUSION}

The COVID 19 pandemic presented all of us with the acid tests comprising of the following triad:

1. evaluation of our standards of care and healthcare system preparedness and capacity

2. the strength of our social capital and

3. the effectiveness of the whole of government and whole of nation approaches in each of our countries.

There were numerous ethical issues on hand, with many challenging decisions that had to be made. This pandemic also reinforces the importance of the concept of 'resilience' or what many would call 'bouncing back'. During COVID 19, we saw resilience as the capacity and capability of our healthcare system and infrastructure in absorbing an upsurge. This calls for pre-pandemic preparedness, planning and coordination; not forgetting to include all the potential ethical dilemmas that could surface. Resilience is also about the ability of the community to respond proactively to changes used to mitigate the crisis. In the end, every country, every institution and every HCW is looking for the best way for society to treat unforeseeable threats and future crisis.

\section{REFERENCES}

[1] Emanuel EJ, Persad G, Upshur R, Thome B, Parker M, Glickman A et al. Fair allocation of scarce medical resources in the time of COVID 19. New Engl Journal of Med 2020; Mar 23. Doi: 10.1056NEJMsb2005114

[2] Kotzee B, Ignatowicz A, Thomas A. Virtue in medical practice: An exploratory study. HEC Forum 2017; 29(1): 1-19

[3] Ng EST, Thambyah PA. The ethics of responding to a novel pandemic. Ann Acad of Med Singapore2011; 40:30-35

[4] Morens DM, Folkers GK, Fauci AS. What is a pandemic? J Infect Dis 2009; 200:1018-1021

[5] Sim K, Chua HC. The psychological impact of SARS: a matter of heart and mind. CMAJ 2004; 170:811-812

[6] Bayer R, Fairchild AL. The genesis of public health ethics. Bioethics 2004; 18(6): 473-492 
Through the Ethical Lenses: There is Really More than Meets the Eye with Covid-19

[7] Evans NG, Hills K, Levine AC. How should the WHO guide access and sharing during infectious diseases outbreaks? AMA Journal of Ethics 2020; 22(1): E28-35

[8] Emanuel EJ. The lessons of SARS. Annals of Intern Med 2003; 129: 589-591

[9] Clark CC. Trust in Medicine. J Med Philos 2002; 27: $11-29$

[10] Gostin L. Public health strategies for pandemic influenza: Ethics and the Law. JAMA 2006; 295:1700-1704

[11] Ruderman C, Tracy CS, Bensimon CM, Bernstein M, Hawryluck L, Shaul RZ et al. On pandemics and the duty to care: whose duty, who cares? BMC Medical Ethics2006; 7: 5. Doi: 10.1186/14726939-7-5

[12] Thompson AK, Faith K, Gibson JL, Upshur RE. Pandemic influenza preparedness: an ethical framework to guide decision making. BMC Med Ethics 2006; 7:E12

[13] Carson R. Balancing loyalties or splitting the difference? Acad Med 2000; 75(50; 443-444

[14] Simonds AK, Sokol DK. Lives on the line. Ethics and practicalities of the duty of care in pandemics and diseases. Eur Resp Journal 2009; 34:303309

[15] Reid L. Diminishing returns? Risks and the duty of care in the SARS epidemic. Bioethics 2005; 19:348-361

[16] Dingwall K, Hoffman LM, Staniland K. Introduction: Why a sociology of pandemic? Sociology of Health and Illness 2013; 35(2): 167-175

[17] Hsin DH, Macer DR. Heroes of SARS: professional roles and ethics of healthcare workers. J Infect 2004; 49: 210-215

[18] Ovadia KL, Gazit I, Silner D, Kagan I. Better late than never: a re-examination of ethical dilemma in coping with SARs. Hosp Infect 2005; 61:75-79

[19] Evans EL, London AJ. Equipose and the criteria for reasonable action. Journal of Law, Medicine and Ethics 2006; 34: 441-450

[20] Wynia MK. Public health principlism: the precautionary principle and beyond. The Am J of Bioethics 2005; 5(3): 3-4

[21] White DB, Katz MH, Luce JM, Lo B. Who should receive life support during a public health emergency? Using ethical principles to improve allocation decision. Ann Intern Med 2009; 150(2): 132-138

[22] Melnychuk RM, Kenny NP. Pandemic Triage: the ethical challenge. CMAJ 2006; 175:1393-1394

[23] Penciner R. Clinical teaching in a busy ED: Strategies for success. CJEM 2002; 4(4);286-288

[24] Torda A. How far does a doctor's duty of care go? Intern Med J 2005; 35(5): 295-296

[25] Cookson R, Dolan P. Principles of justice in healthcare. Journal of Medical Ethics 2000; 26:323-329

[26] Mah M, Myers G. Towardsa socio-ethical approach to behavioural change. Annals of Emerg Med 2006; 34(2): 73-79

[27] Mare S, Sutjita M, Rajagopalan S. Bioterrorism, Bioethics and the Emergency Physician. Topics in Emergency Medicine. Ethics in Emergency Dept 2004; 26(1): 44-48

[28] Sokol DK. Virulent Epidemic and scope of healthcare workers duty of care. Emerging Infect Dis 2001; 12(8): 1238-1241

[29] Rhodes R. Justice in Medicine and Public Health. Cambridge Quarterly Healthcare Ethics 2005; $14(10 ; 13-26$

[30] Levine AC. Academics are from Mars and Humanitarians are from Venus: finding common ground to improve research during humanitarian emergencies. Clin Trials 2016; 13(1); 79-82

[31] Vawter DE, Garrett JE, Prehn AW, Gervais KG. healthcare workers willingness to work in a pandemic. Am J of Bioethics2008; 8(8); 21-23

[32] Iserson KV, Heine CE, Levkin GL, Moskop JC, Baruch J, Aswegan AL. Fighter flight: the ethics of emergency physician disaster response. Annals Emerg Med 2008; 51(4): 345-353

[33] Berlinger N, Wymia M, Powell T, Hester M, Milliken A, Fabi R, Coha F et al. Ethical framework 
Through the Ethical Lenses: There is Really More than Meets the Eye with Covid-19

for healthcare institutions responding to novel COVID 19. The Hastings Centre, 16 March 2020. Available at https://www.thehastingscenter. org/ethicalframeworkcovid19 (last accessed on 14 April 2020)

[34] Bernstein D. Rethinking the physician's duty in disaster care. Am Med Assn J Ethics 2010; 12(6): 460-465

[35] Moskop JC, Iserson KV. Triage in Medicine Part
II: Underlying values and principles. Ann Emerg Med 2007; 49(3); 282-287

[36] UN Committee on Human Rights. The Siracusa Principle on the limitations and derogation provisions in The International Convenant on Civil and Political rights. 28 Sept 1984; E/ CN.4/1985/4. Available at http://www.unhcr. org/refworld/docid/4672bc122.html(last accessed on 14 April 2020)

Citation: Fatimah Binte Abdul Lateefahit. Through the Ethical Lenses: There is Really More than Meets the Eye with Covid-19. Archives of Emergency Medicine and Intensive Care. 2020; 3(1): 06-16.

Copyright: (C) 2020 Fatimah Binte Abdul Lateefahit. This is an open access article distributed under the Creative Commons Attribution License, which permits unrestricted use, distribution, and reproduction in any medium, provided the original work is properly cited. 